\title{
CONDIÇÕES QUE PROMOVEM O APRIMORAMENTO PROFISSIONAL E COMPORTAMENTOS RELACIONADOS À MUDANÇA CLÍNICA: ATRIBUIÇÕES DE TERAPEUTAS COMPORTAMENTAIS EXPERIENTES E NÃO EXPERIENTES
}

\author{
COMPARISON OF ATRIBUITIONS DONE BY \\ EXPERIENCED AND NOVICE BEHAVIORAL THERAPISTS
}

Luciana Ferreira ALVAREZ ${ }^{1}$

Jocelaine Martins da SILVEIRA ${ }^{2}$

\begin{abstract}
RESUMO
Comparação de atribuições feitas por psicoterapeutas comportamentais experientes e não experientes $(N=41)$ sobre condições que promovem o aprimoramento profissional. Por meio de um questionário, 41 psicoterapeutas responderam a questões sobre condições que facilitam o aprimoramento da prática clínica e sobre o repertório comportamental do psicoterapeuta relacionado com a mudança clínica. Uma lista de categorias de comportamentos de terapeutas relacionados à mudança clínica é apresentada. As condições encontradas como relevantes para o aprimoramento da prática clínica foram comparadas em termos da experiência do terapeuta. Elas parecem estar relacionadas às situações presentes no contato direto do terapeuta com seus clientes (experiência - número de casos atendidos) e condições de aprimoramento técnico (formação acadêmica e atualização bibliográfica).
\end{abstract}

Palavras-chave: Prática clínica, psicoterapeutas experientes, psicoterapeutas não experientes.

\begin{abstract}
By way of a questionnaire behavioral therapists $(N=41)$ answered to questions about conditions that favor the improvement of the clinical practice and the behavioral repertory of the psychotherpist related to the

(1) Docente da Universidade Estadual de Londrina - PR . R.Sebastião Chapp Galindo, 50, bloco II, apt 603. Bairro

(2) Docente da Universidade Estadual de Londrina - PR. Av. Japão, 266, Jardim Cláudia. CEP 86050-170 - Londrina PR.
\end{abstract} Jerusalém, cep 86051-570. E-mail: lalvarez@uel.br. E-mail: jsilveira@sercomtel.com.br 


\begin{abstract}
clinical change. The important conditions for the improvement of the clinical practice where debated, which appeared to be related to the circumstances present in the psychotherapist direct contact with their clients (experience - number of cases) and quality of technical improvement (academic develpment and being update).
\end{abstract}

Key-words: Clinical practice, experienced psychotherapists, novice psychotherapists.

Uma boa parte dos estudos sobre Psicoterapia investiga a eficácia de tratamentos ou a apropriação de determinadas intervenções clínicas para psicopatologias específicas. Menos freqüentemente, encontram-se investigações dos eventos que ocorrem durante as sessões de psicoterapia, considerada, nestes casos, uma importante condição natural a ser examinada. Os pesquisadores que desenvolvem estudos desse tipo estão preocupados em esclarecer detalhes da interação que se estabelece entre o cliente e o psicoterapeuta antes que se proceda qualquer avaliação da eficácia dos procedimentos empregados (BANACO, 1996; CZOGALIK e RUSSELL, 1995; GARDNER, 1993; GREENBERG e NEWMAN, 1996; KOLDEN, 1996; RICE e GREENBERG, 1984; SEXTON, HEMBRE, KVARME, 1996; SHAFFER, 1982; RICHARDS, LONBORG, 1996; SILVEIRA, 1997; SILVEIRA e KERBAUY, 2000).

GAVINO (1996) relaciona, pelo menos, três principais grupos de pesquisas que estudam a mudança comportamental durante as sessões de terapia. São eles: 1) estudos que enfatizam a abordagem direta do problema, dedicando-se à criação de técnicas e estratégias direcionadas ao tratamento da patologia (comportamento problema); 2) estudos diferenciais dos pacotes terapêuticos e 3) pesquisas sobre variáveis que estão presentes no processo terapêutico. Nesse último grupo, distinguem-se três tipos principais de estudos. Aqueles que investigam: a) as variáveis relacionadas ao terapeuta (habilidades ou características pessoais), b) as variáveis relacionadas ao cliente e, finalmente, c) a interação entre ambos.

\subsection{0 terapeuta como uma variável no estudo da Psicoterapia:}

Sabidamente, terapeutas treinados ajudam o cliente significativamente mais, se comparados a profissionais não treinados (GOLD e DOLE, 1989). Entretanto, o comportamento do terapeuta permanece relativamente pouco estudado, o que levou alguns estudiosos a se referirem a ele como uma "variável negligenciada" na investigação dos resultados terapêuticos (BEUTLER, 1997; GARFIELD, 1997).

Características do terapeuta foram avaliadas por alguns pesquisadores, os quais as relacionaram com os resultados do tratamento e/ou com a satisfação dos clientes (VASCO e DRYDEN, 1994). PROPST, PARIS e ROSBERGER (1994) investigaram o efeito da experiência do terapeuta, do funcionamento (desempenhos) e do diagnóstico clínico nos resultados da psicoterapia breve. Assim, foram diagnosticados 123 pacientes, os quais foram atendidos por um psiquiatra, um residente em psiquiatria, um residente em prática com famílias ou por um estudante. Todos os sujeitos apresentaram melhoras significantes de acordo com avaliações de linhas de base. Tais melhoras foram mantidas pelo menos por um período de seis meses de follow-up. O estudo verificou que nem o tipo de terapeuta e tampouco a categoria diagnóstica (ajustamento, humor, ansiedade ou desordens de personalidade) apresentaram correlação 
com os resultados da terapia. Por outro lado, a satisfação do cliente com a terapia esteve altamente relacionada com o aumento do funcionamento (desempenhos) e decréscimo na severidade dos sintomas. O número de sessões de terapia esteve modestamente relacionado ao resultado e com a satisfação do cliente. Por tratar-se de um estudo correlacional, permanece a questão: a melhora clínica teria provocado a satisfação do cliente ou sua própria motivação (satisfação) com a terapia facilitou mudanças clínicas?

A aliança terapêutica é um conceito derivado da orientação psicodinâmica e fora conceituado originalmente por BORDIN (1979), consistindo na existência harmoniosa de três componentes: o vínculo que se estabelece entre o terapeuta e seu cliente, os objetivos e as tarefas da terapia. Segundo DIGIUSPPE, LINSCOTT e JILTON (1996) a aliança terapêutica correlaciona-se positivamente com os resultados da terapia e independe da orientação teórica do psicoterapeuta.

BOOTZIN e RUGGILL (1988) observaram que, possivelmente, a habilidade do clínico em estabelecer a aliança terapêutica não seja uma condição suficiente para a promoção de resultados terapêuticos, mas, é extremamente importante, já que por meio dela a eficácia das técnicas terapêuticas aplicadas é potencializada.

MAHONEY e ALVAREZ (1998) examinaram a vida pessoal de 206 psicoterapeutas por meio de um inventário. Os resultados demonstram que a maioria deles considerou-se feliz e saudável e que experimentou alegrias na infância. As situações mais difíceis na psicoterapia, segundo os psicoterapeutas são: a resistência dos clientes ao tratamento, tentativas de suicídio, comportamento paranóide e delirante, abandono da terapia e raiva expressa contra o terapeuta. As preocupações pessoais mais comuns indicadas foram insegurança financeira, ansiedade e exaustão física. As estratégias de enfrentamento adotadas pelos profissionais incluem: socialização, assistir televisão, leituras prazerosas e exercícios. A maioria dos sujeitos relatou já haver se submetido à psicoterapia, considerando isso uma experiência positiva. Os efeitos da prática clínica na vida pessoal dos psicoterapeutas, de modo geral, foram considerados positivos.

E ao invés? Isto é, de que modo a vida pessoal do terapeuta teria efeitos na prática clínica? WILLIAMS, COYLE E LYONS (1999) aplicaram um questionário a psicoterapeutas $(\mathrm{N}=192)$, para investigar como viam suas terapias pessoais. Os resultados indicaram que $88 \%$ eram favoráveis à terapia pessoal como um requisito para o treino profissional. $A$ maioria, considerou o resultado e o processo de sua terapia positivos.

\subsection{Análise Comportamental e o papel do psicoterapeuta:}

Há 20 anos, pelo menos, estudiosos vêm preconizando o emprego da Análise Comportamental no estudo da situação clínica em Psicoterapia. Segundo FERSTER (1979), a linguagem comportamental apresenta vantagens no trabalho clínico, posto que o princípio básico de uma descrição comportamental é uma separação da conduta humana no ato em si e nas alterações no ambiente que ele produz. Em um capítulo intitulado "Psicoterapia do ponto de vista de um Behaviorista", FERSTER indica a interação que se estabelece entre o terapeuta e o cliente como o elemento por meio do qual acontece a mudança terapêutica. O autor propõe que sejam consideradas as alterações no comportamento do próprio terapeuta na medida em que interage com seu cliente. As recomendações de FERSTER foram notadamente atendidas no final da década de oitenta e na década de 90 por analistas comportamentais. CORDOVA e KOHLENBERG (1994) preocuparam-se com o papel da relação terapêutica, utilizando-a 
como instrumento para promoção da mudança clínica.

Admitindo as proposições de FERSTER, é razoável supor que clientes também modelem comportamentos em seus terapeutas (além da relação inversa). Portanto, psicoterapeutas mais experientes tendem a apresentar comportamentos mantidos pelo sucesso na manipulação do comportamento de seus clientes (GUILLARDI, 1997). A identificação desse repertório contribuirá para a compreensão da mudança terapêutica, assim como para o planejamento da formação de psicoterapeutas.

A despeito de se dispor de dados e conceitos como os acima descritos, pouco se tem avançado na identificação de: a) condições que facilitem o treino de terapeutas, b) habilidades básicas a serem treinadas na formação de terapeutas e c) de distinções entre os desempenhos de terapeutas proficientes (experientes) e terapeutas não experientes ou iniciantes.

\subsection{Como se comportam os psicotera- peutas proficientes?}

Um caminho para a identificação dos itens acima parece ser a investigação do repertório de terapeutas experientes ou a comparação desse repertório com o de terapeutas iniciantes. Seria necessário conhecer também as atribuições feitas pelos psicoterapeutas experientes de condições que promoveram seu aprimoramento profissional, assim como de comportamentos aos quais creditam as mudanças desejáveis clinicamente no comportamento de seus clientes.

Um estudo de DIAMONDE e MARTINOVICH (1997) constatou que à medida que os terapeutas tornam-se mais experientes eles estão menos inclinados a fazer intervenções afetivas em fases precoces do tratamento e tornam-se mais propensos a usá-las mais tarde no curso da psicoterapia. Terapeutas relativamente menos experientes estiveram mais inclinados a demonstrar consistência no uso de suas intervenções em todas as sessões. Isso sugere que os terapeutas mais experientes mantenham-se sob o controle do próprio relacionamento com o cliente, enquanto que os iniciantes apresentam um padrão de intervenção consistente e independe do aumento de intimidade no relacionamento com o cliente.

No Brasil, BANACO (1996) identificou diferenças significativas entre as intervenções durante as sessões de atendimento, de terapeuta experientes e terapeutas inexperientes. Os terapeutas experientes apresentaram mais intervenções durante a sessão e sabiam explicá-las claramente. Por outro lado, terapeutas inexperientes ficavam sob o controle de regras envolvidas no aprendizado de atender e apresentaram ausência total de verbalizações sobre autopercepção.

Portanto, a literatura sugere diferenças no comportamento de terapeutas experientes e terapeutas iniciantes. De quais condições derivariam, predominantemente, tais diferenças? Um estudo de LYMAN, STORM E YORK (1995) sugere que a mera sucessão de experiências na vida pessoal do terapeuta não é uma explicação suficiente. Os referidos pesquisadores não encontraram correlações positivas entre atribuição de eficiência que clientes faziam em relação aos psicoterapeutas e a quantidade de experiências na vida do profissional.

Nesse ponto, convém examinar algumas recomendações dadas por um psicoterapeuta com mais de 50 anos de experiência - W. M. HAAS (1997), que se designa "um veterano". Os princípios que, segundo ele, podem contribuir para a obtenção de resultados terapêuticos são: 1) conhecer-se, 2) proteger sua privacidade e seus princípios, 3) desenvolver seu próprio estilo, 4) utilizar a experiência do aqui-agora, 5) sintonizar-se com sua criança interna; 6) apreciar opções ecléticas, 7) ajudar clientes a irem além de 
sua raiva, 8) encarar a morte e 9) gostar de sua atuação.

BANACO (1996) acrescenta um outro requisito para uma atuação satisfatória do terapeuta: ter cultura geral para melhor decodificar metáforas apresentadas no relato do cliente. Tais metáforas seriam as descrições do cliente no contexto em que seu comportamento ocorre.

\subsection{A formação de psicoterapeutas:}

Sem conhecer o suficiente as habilidades de psicoterapeutas proficientes e sua interferência como uma variável potencial na produção dos resultados terapêuticos, a formação de psicoterapeutas, em geral, tem se fundamentado apenas em dados que se tornaram mais tradicionais e comumente conhecidos. O estudo pioneiro de ROGERS (1961), o qual descreve características desejáveis em um psicoterapeuta tais como empatia, crença na vocação humana para o crescimento, habilidades para aceitar incondicionalmente e fornecer feedbacks reflexivos (sobre emoções); ainda é um importante referencial para a formação de inúmeros psicoterapeutas.

$\mathrm{Na}$ década de setenta e início da seguinte, encontram-se vários estudos sobre a eficácia relativa de métodos e recursos para se treinar terapeutas comportamentais (BAILEY, DEARDORFF, e NAY, 1977; BOUCHARD, WRIGHT, MATHIEU, LALONDE, BERGERON e TOUPIN, 1980; WRIGHT e MATHIEU, 1981). Nessa ocasião, avaliou-se, por exemplo, o treino de respostas do terapeuta de levar o cliente a contactar contingências, a CTRL, fundamentada na conceituação skinneriana do comportamento verbal (EISENBERG e DELANEY, 1970; ROBINSON, FROEHLE e KURPIUS, 1979). Em meados da década de oitenta em diante, já pode ser notada uma preocupação em sistematizar os dados de pesquisa obtidos (BERNSTEIN, 1982 e BOOTZIN e RUGGILL, 1988).
O interesse pelo assunto continuou crescendo nos últimos anos (MCLENNAN, 1994; STEIN e LAMBERT, 1995; PEKARIK, 1994; SILVARES e GÔNGORA, 1998; BANACO, 1995; 1996), mas, ainda há muitas questões sem resposta sobre a formação de psicoterapeutas. Quais habilidades são tipicamente notadas no repertório comportamental de psicoterapeutas experientes, cujo treino seja viável para terapeutas iniciantes?

Segundo FOLLETE, CALLAGHAN e GLENN (1995) são ainda limitados os dados sobre como treinar psicoterapeutas. Esses autores descreveram uma metodologia de treinamento baseada na modelagem contingente, no feedback em vídeo e no aumento da responsividade nas interações terapeuta-cliente, buscando superar uma formação baseada meramente em instruções diretas no governo por regras.

MAHONEY (1998) relaciona os seguintes temas, considerados por ele, essenciais no treino de psicoterapeutas: 1) auto-conhecimento; 2) relacionamento humano; 3) compaixão; 4) filosofia; 5) habilidades de sobrevivência e enfrentamento; 6 ) valores e 7) qualidade de vida. Segundo o autor, tais habilidades não são adequadamente ensinadas em cursos acadêmicos formais e nos modelos tradicionais de ensino. Assim, segundo MAHONEY, mais importante que o conteúdo dos cursos e do curriculum é o contexto humano e a atmosfera de aprendizagem nos programas de treinamento. Tais contextos e atmosferas deveriam encorajar o desenvolvimento dos temas acima citados.

A relevância da supervisão de práticas em psicoterapia vêm sendo um aspecto recentemente estudado no âmbito da formação de psicoterapeutas (LAMBERT, OGLES, 1997; HOLLOWAY, NEUFELD, 1995 e ASHURST, 1993). Um estudo de KAUFMAN e KORNER (1997) investigou percepções de 53 membros de uma associação de profissionais, com idades variando de 25 a 75 anos acerca de sua 
formação. Eles foram solicitados a avaliar a contribuição, se positiva ou negativa, de instrutores da graduação e supervisores de habilidades terapêuticas. O estudo verificou que os supervisores parecem ter exercido um papel importante na formação daqueles profissionais e que os aprendizes tendem a adotar condutas que julgam importantes em seus treinadores.

Em suma, ainda são modestos os dados acerca de como psicoterapeutas intervém durante as sessões de terapia e de como essas variáveis potenciais influenciam os resultados terapêuticos. Supõe-se que psicoterapeutas experientes tenham seus comportamentos modelados na interação com seus clientes. Desse modo, a identificação de comportamentos que julgam estar relacionados à mudança clínica, pode contribuir fornecendo subsídios relevantes para a formação de psicoterapeutas.

Assim, o presente estudo se propõe a: 1) identificar condições facilitadoras do aprimoramento da prática clínica, segundo a atribuição de terapeutas experientes (TE) e não experientes (TNE) relativamente, assim como os comportamentos que os profissionais julgam estar relacionados à mudança clínica e 2) comparar as atribuições apresentadas pelos TE e TNE, verificando (comparativamente) quais condições são indicadas como facilitadoras do aprimoramento profissional.

\section{MÉTODO}

\section{Participantes}

Responderam ao questionário, 41 psicoterapeutas comportamentais ( $N=41)$, com tempo mínimo de prática clínica de dois anos e pelo menos, um caso clínico concluído. Todos atuantes em clínicas privadas e/ou clínicas-escola da cidade de Londrina - PR.

\section{Procedimento}

Seleção da amostra: os psicoterapeutas comportamentais que participaram do estudo tiveram sua orientação teórica e o local de trabalho identificados por meio de uma consulta feita previamente na sub-sede do Conselho Regional de Psicologia PR. A identificação dos componentes da amostra como "experientes" ou "não experiêntes" relativamente foi realizada a posteriori.

Distinguiram-se dois grupos da amostra. Os sujeitos cuja experiência clínica abrangeu até 20 casos concluídos foram considerados Terapeutas não Experientes (TNE) e os sujeitos que atenderam mais de 20 casos foram considerados Terapeutas Experientes (TE).

Aplicou-se um questionário (Anexo 1) previamente testado em um estudo piloto em 12 terapeutas comportamentais. Durante a aplicação do instrumento, os psicoterapeutas foram solicitados a responder ao questionário para colaborarem com um estudo sobre "a atuação de psicoterapeutas". As demais informações sobre o estudo constam no início da página do questionário.

Por meio do instrumento, os psicoterapeutas foram solicitados a responder a perguntas sobre 1) o tempo e número de casos atendidos; 2) indicação de condições facilitadoras do aprimoramento profissional e 3) indicação de comportamentos que se relacionam com a mudança clínica do cliente durante as sessões.

A sistematização dos dados constou de avaliação percentual das respostas para as questões que compuseram os dados referentes aos itens um e dois. Os dados foram analisados pelo Núcleo de Processamento de Dados (NPD), da Universidade Estadual de Londrina, por meio do Statics Analysis System, SAS.

As respostas que compuseram o último item do questionário foram agrupadas em dez categorias. 
I - Incluiram-se nessa categoria, termos como "confrontar cliente no momento adequadro"; "bloquear esquiva" ou outras respostas referentes ao tema.

II - Foram categorizadas respostas como: "ser empático com o cliente"; "empatia" ; "valorizar e respeitar a pessoa do cliente" etc.

III - Foram incluídas nessa categoria, expressões como: "reforçar comportamentos do cliente na sessão", "reforçamento natural"; "reforçamento de comportamentos adequados" e "reforçamento diferencial".

IV - Foram considerados nesse ítem, respostas como: "o terapeuta discriminanar as reações privadas relacionadas aos comportamentos emitidos na sessão pelo cliente", "terapeuta conhecer-se bem", "terapeuta ter autoconhecimento", "terapeuta identificar suas emoções relacionadas a sua história de vida, provocadas pelo cliente".

V - Nessa categoria, incluíram-se respostas cujo tema se relacionou a Análise Funcional do comportamento do cliente.

VI - Incluiram-se nessa categoria, respostas relacionadas á verificação feita pelo terapeutas da fala do cliente.

VII - Encontram-se nessa categoria, expressões sobre o tema "não reforçar o comportamento-problema do cliente".

VIII - Foram considerados nessa categoria, termos como: "ensinar o cliente a emitir comportamentos que levem ao enfrentamento do problema", "ajudar o cliente a pensar em estratégias para lidar com suas dificuldades".

IX - Inclui-se, nessa categoria, uma questão não respondida.

X - Nessa categoria, encontram-se expressões como: "dar informações ao cliente sobre suas dificuldades", "falar numa linguagem acessível ao contexto do cliente" entre outras excluídas das categorias anteriores.
A análise dos dados baseou-se na interpretação das respostas ao questionário, comparando as indicações de terapeutas experientes (TE) e não experientes (TNE) quanto às condições que facilitam o aprimoramento da prática clínica.

\section{Local}

Os questionários foram aplicados em clínicas psicológicas particulares e de clínicasescola, cujos profissionais eram de orientação comportamental, na cidade de Londrina - PR .

\section{RESULTADOS}

Os profissionais que responderam o questionário apresentaram idades variando de 22 a 52 anos, o único sujeito do sexo masculino tinha 29 anos. O tempo de experiência clínica dos profissionais distribuiu-se em três grandes grupos, 36,6\% tinham até dois anos de atuação na área clínica; 29,3\% tinham de dois anos a cinco e $34,1 \%$, mais de cinco anos de trabalho clínico.

Quanto ao número de casos atendidos pelos sujeitos durante o tempo de atuação clínica, $17 \%$ atenderam até cinco casos, 29,3\% atenderam entre 20 a 40 casos, $22 \%$ atenderam entre 20 a 40 casos e finalmente $31,7 \%$ dos profissionais tinham atendido a mais de 40 casos.

Os comportamentos indicados pelos terapeutas foram agrupados nas seguintes categorias e respectivos temas: I - Bloquear esquiva do cliente; II - Aceitação e Empatia; III - Modelagem direta na sessão; IV - Autoconhecimento do terapeuta; V - Ensinar o cliente a fazer Análise Funcional; VI - Checar a compreensão de verbalizações do cliente; VII - Evitar reforçar o problema trazido pelo cliente; VIII - Ensinar o cliente a desenvolver estratégias de enfrentamento do problema; IX - Não responderam e X - Outros. Sendo que os comportamentos predominantemente 
citados por TEs e TNEs compuseram as categorias: "ensinar o cliente a analisar funcionalmente seus comportamentos" (36 menções); "modelagem direta na sessão" (28 vezes citado) e "aceitação/empatia" (citado 25 vezes).

Quanto às condições que facilitam o aprimoramento profissional, o percentual de desprezo do item $E$ (experiência profissional concomitante a prática clínica) referente à sexta questão do questionário, foi de quase metade da amostra, 46,3\%, enquanto que o itens G, D, e F (interação com os clientes, continuidade da formação acadêmica, atualização bibliográfica) obtiveram os maiores percentuais de escolha pelos sujeitos somando-se suas escolhas em $1^{\circ}$ e $2^{\circ}$ lugar, $(43,9 \%, 41,5 \%$ e $36,6 \%)$ respectivamente. Os itens G, D, e B (interação com os clientes, continuidade da formação acadêmica, submeter o caso a supervisão ) obtiveram os percentuais de escolha em $1^{0}$ - lugar mais elevados.

Os TEs apresentaram como itens mais escolhidos como condições para o aprimoramento da prática clínica: $1^{\circ}$ ) interação com os clientes, $2^{\circ}$ ) submeter o caso a supervisão clínica e $3^{\circ}$ ) continuidade da formação acadêmica. Os terapeutas pouco experientes apresentaram como ítens mais escolhidos: $1^{\circ}$ ) continuidade da formação acadêmica, $2^{\circ}$ ) interação com os clientes e $3^{\circ}$ ) submeter o caso a supervisão clínica. O item E (experiência profissional concomitante a prática clínica) foi o menos escolhido; para os TNE e o item H (submeter-se à psicoterapia) foi o menos indicado pelos TE.

Os TEs escolheram os itens de $1^{\circ}$ ao $5^{\circ}$ lugares na respectiva ordem: interação com os clientes, submeter o caso a supervisão clínica, continuação da formação acadêmica, atualização bibliográfica relacionada a prática clínica e participação em eventos científicos. Já os TNEs assim organizaram os itens: primeiro - continuação da formação acadêmica; segundo - interação com os clientes; terceiro - submeter o caso a supervisão clínica; quarto - atualização bibliográfica relacionada a prática clínica e quinto - sua própria história de vida.

\section{DISCUSSÃO}

É preciso considerar, preliminarmente, que todos os dados ficam subordinados ao critério de "experiência", no momento em que se estabeleceram as categorias TE e TNE, com base na conclusão bem sucedida de casos clínicos. O critério pode se relacionar, de algum modo, com a escolha de condições que facilitam o aprimoramento profissional, já que um terapeuta jovem poderia ser incluído na categoria TE e vice-versa. O critério adotado admitiu como "experiente" o terapeuta que foi mais vezes exposto a condições de modelagem na interação com o cliente.

A amostra dividiu-se em 46,3\% de profissionais não experientes (TNE) e 53,7\% de profissionais experientes. A maioria desses profissionais considerou relevante para o aprimoramento de sua prática clínica a própria interação com seus clientes durante as sessões psicoterápicas, assim como a continuidade da formação acadêmica (prioritariamente cursos de Especialização) e atualização bibliográfica referente à atuação clínica, respectivamente. As condições mais freqüentemente consideradas irrelevantes para o aprimoramento profissional foram "desenvolvimento de atividade profissional concomitantemente à atuação clínica"; "submeter-se à psicoterapia" e "eventos da própria história de vida do terapeuta".

Esses dados sugerem que as condições favorecedoras do aprimoramento profissional relacionem-se, predominantemente, com a interação que se estabelece entre o psicoterapeuta e seus clientes; corroborando as proposições de FERSTER (1979) segundo as quais o comportamento do terapeuta está sujeito à modelagem na interação com o 
cliente. Logo, psicoterapeutas mais experientes tendem a apresentar comportamentos mantidos pela interação com seus clientes (GUILHARDI, 1997 e BANACO, 1997).

O desenvolvimento técnico do psicoterapeuta foi também uma condição freqüentemente citada pelos profissionais, uma vez que priorizaram a continuidade da formação acadêmica e atualização bibliográfica relacionada à prática. Isso indica que o treino e a técnica dos profissionais devem interferir no sucesso da terapia, conforme já mencionado na literatura por GOLD e DOLE (1989).

Por outro lado, a vida pessoal dos psicoterapeutas (incluindo eventos da história de vida deles) ou mesmo experiências profissionais outras, concomitantes à clínica, foram condições consideradas por eles pouco relevantes para o aprimoramento profissional. Este dado parece estar de acordo com LYMAN, STORM e YORK (1995) que não encontraram correlações positivas entre a satisfação dos clientes e a quantidade de experiências na vida pessoal dos terapeutas.

Curiosamente, as três condições mais relevantes indicadas por TE e TNE foram as mesmas, embora estivessem em ordens diferentes de prioridade. São elas: "submeter o caso à supervisão clínica"; "continuidade da formação acadêmica" e "interação com os clientes".

Quanto às categorias de comportamentos relacionados à mudança clínica. As mais freqüentemente citadas foram: "ensinar o cliente a analisar funcionalmente seus comportamentos"; "modelagem direta na sessão" e "aceitação/empatia", respectivamente. Elas parecem requerer dos terapeutas várias habilidades de relacionamento interpessoal.

Estudos futuros são necessários para propor e testar metodologias de treinamento de psicoterapeutas, de modo que fiquem sensíveis às contingências da relação com o cliente - a exemplo de uma proposta de FOLLETE, CALLAGHAN e GLENN (1995). Além disso, mais estudos comparativos do desempenho e/ou características de terapeutas experientes e não experientes deverão subsidiar a formação de futuros psicoterapeutas.

\section{REFERÊNCIAS}

ARANHA, M.S.F. A interação social e Desenvolvimento Humano. Temas em Psicologia, São Paulo, n.3, pp. 19 - 48, 1993.

BANACO, R.A. O Desenvolvimento pessoal de terapeuta: Análises informais de um formador em psicoterapia comportamental. Torre de Babel: Reflexões e Pesquisa em Psicologia, Londrina, v.3, pp.27 - 41, 1996.

.O impacto do atendimento sobre a pessoa do terapeuta 2 : experências de vida. $1^{\circ}$ ed., pp. 174 - 181, São Paulo. ARBytes., 1997.

BEUTLER, L.E. The psychotherapist as a neglected vairable in psychotherapy: an illustration by reference to the role of therapist experience \& training. Clinical Psychology Science and Practice, v. 4 (1), p. 44-52, 1997.

DIAMOND, J; MARTINOVICH, Z. The impact of training on therapists use of interventions. Psychotherapy - Research, v. 7 ( 3 ), p. 239 - 247, 1997.

DIGIUSEPPE, R; LINSCOTT, J; JILTON, R. Developing the therapeutic alliance in child - adolescent psychotherapy. Applied and Preventive Psychology. v.5, p. 85 - 100, 1996.

FERSTER,C.B. Et al. Princípios do Comportamento. $2^{\circ}$ ed. São Paulo: Global, 1982. 
FOlLETTE, W. C; CALLAGHAN, G. M: Do as I do , not as I say: A behavior analytic approach to supervision. Professional Psychology: Research and Pratice. V. 26 (4), p. 413 - 421, 1995

GARFIELD, S. L. The psychotherapist as a neglected vairable in psychotherapy research. Clinical Psychology Science and Practice, v. 4 (1), p. 40-43, 1997.

GOLD, J ; DOLE, A. A. Professional psychotherapists vsnon psychotherapists: Thought processes, verbal behavior, and clients satisfaction. Psychological Reports, v. 65, p. 611 - 620, 1989.

GOLDFRIED, M. R ; RAVE, P. J; LOUIS, G. C. Therapeutic focus in significant sessions of master therapists: A Comparison of Cognitive - Behavioral and Psychodynamic Interpersonal Intervetions. Journal of Consulting and Clinical Psychology. V. 66, no $5,803-810,1998$.

GUILHARDI, H. Com que contingências o terapeuta trabalha em sua atuação clínica. $1^{\circ}$ ed. São Paulo: ARBytes, p. 322 - 337 , 1997.

HAAS, W. M . Psychoterapy: Some guiding principles. American Journal of Psychotherapy. v. 51 ( 4 ) , p. 593 - 606, 1997.

KAUFMAN, M ; KORNER, S. Psychotherapist $S^{\prime}$ perceptions of their trainers and training emphases. Clinical - Supervisor. v. 16 (1), p. 97 - 115, 1997.

LYMAN, B. J; STORM, C. L; YORK, C. D. Rethinking assumptions about trainees' life experience. Journal of Marital and Family Therapy. V. 21 ( 2 ), p. 193 - 203, 1995.

MAHONEY, M. J.. Essencial themes in the training of psychoterapists. Psychoterapy in Private Practice. v. 17 ( 1 ), p. 43 - 59, 1998.
MAHONEY, M. J; ALVAREZ, F. La vida personal del psicoterapeuta/ The personal life of the psychotherapist. Avances en Psicologia Clínica Latinoamericana. v. 16, p. 9 - 22, 1998.

PROPST, A.; PARIS \& J. ROSBERGER, Z. Do therapist experience, diagnosis and functional level predict outcome in short term psychotherapy. Canadian Journal of Psychiatry, v. 39 (3)p 168-176, 1994.

ROGERS, C. Terapia Centrada no Cliente. São Paulo: Martins Fontes, 1961.

ROSENFARB, I. S. A Behavior Analytic Interpretation of tht Therapeutic Relationship. The Psychological Record. V. 5, p. 85 - 100, 1996

SEXTON, H.C; HEMBRE, K ; KVARME, G. The Interaction of the Alliance and Therapy Microprocess: A Sequencial Analysis. Journal of Consulting and Clinical Psychology. V. 64, n० 3 , p. 471 - 480. 1996.

SILVARES, E. F de M; GONGÔRA, M. Entrevista clínica inicial. Londrina: EDICON, 1998.

SILVEIRA, J. M. A queixa como condição para análise da interação terapeuta - cliente. São Paulo, 1997. Dissertação ( Mestrado em Psicologia Experimental). Instituto de Psicologia da Universidade de São Paulo).

SILVEIRA, J. M. e KERBAUY, R. R. A interação terapeuta-cliente: uma investigação com base na queixa clínica. Em: R. R. Kerbauy (Org). Sobre o comportamento e cognição: Vol. 5. Conceitos, pesquisa e aplicação. A ênfase no ensinar, na emoção e no questionamento clínico. (pp. 213-221). Santo André: SET, 2000.

STEIN, D. M; LAMBERT, M. J. Graduate training in psychotherapy: Are therapy 
outcomes enhanced? . Journal of Consulting and Clinical Psychology. V. 6(2), p. 182 -196, 1995.

VASCO, A. B; DRYDEN, W. Development of psychotherapist theoretical orientation And clinical practice. British Journal of Guidance and Counselling, v. 22 (3), p. 327-341, 1994.

WILLIAM, F., COYLE, A. E LYONS, E. How counselling psychlogists view their personal therapy. Brithish Journal of Medical Psychology, 72, pp. 545-555, 1999. 